\title{
Expression of arylamine $N$-acetyltransferase in human intestine
}

\author{
D Hickman, J Pope, S D Patil, G Fakis, V Smelt, L A Stanley, M Payton, J D Unadkat, \\ E Sim
}

Department of Pharmaceutics, University of Washington, Seattle, Washington, USA

D Hickman

S D Patil

J D Unadkat

Department of Pharmacology, University of Oxford, Oxford, UK

J Pope

G Fakis

V Smelt

M Payton

E Sim

Department of Pharmaceutical Sciences, De Montfort University, Leicester, UK

L A Stanley

Correspondence to: Professor E Sim

Department of

Pharmacology, University of Oxford, Mansfield Road,

Oxford OX1 3QT, UK.

Accepted for publication 23 September 1997

\begin{abstract}
Background-Arylamine $N$-acetyltransferases in humans (NAT1 and NAT2) catalyse the acetylation of arylamines including food derived heterocyclic arylamine carcinogens. Other substrates include the sulphonamide 5-aminosalicylic acid (5-ASA), which is an NAT1 specific substrate; $N$-acetylation of 5-ASA is a major route of metabolism. NAT1 and NAT2 are both polymorphic.
\end{abstract}

Aims-To investigate NAT expression in apparently healthy human intestines in order to understand the possible role of NAT in colorectal cancer and in the therapeutic response to 5-ASA.

Methods-The intestines of four organ donors were divided into eight sections. DNA was prepared for genotyping NAT1 and $N A T 2$ and enzymic activities of NAT1 and $N A T 2$ were determined in cytosols prepared from each section. Tissue was fixed for immunohistochemistry with specific NAT antibodies. Western blotting was carried out on all samples of cytosol and on homogenates of separated muscle and villi after microdissection.

Results-NAT1 activity of all cytosols was greater than NAT2 activity. NAT1 and NAT2 activities correlated with the genotypes of $N A T 1$ and $N A T 2$ and with the levels of NAT1 staining determined by western blotting. The ratio of NAT1:NAT2 activities showed interindividual variations from 2 to 70 . NAT1 antigenic activity was greater in villi than in muscle. NAT1 was detected along the length of the villi in the small intestine. In colon samples there was less NAT1 at the base of the crypts with intense staining at the tips.

Conclusions-The interindividual variation in NAT1 and NAT 2 in the colon could affect how individuals respond to exposure to specific NAT substrates including carcinogens and 5-ASA.

(Gut 1998;42:402-409)

Keywords: arylamine $\mathrm{N}$-acetyltransferase; 5-aminosalicylate; colorectal cancer; drug metabolism; inflammatory bowel disease; diet

Arylamine $\mathrm{N}$-acetyltransferases metabolise a range of hydrazines and arylamines (see Weber and Hein ${ }^{1}$ for review). The arylamine substrates include environmental carcinogens found in the rubber and dye industry (see Sim et $a l^{2}$ for review) and cigarette smoke. ${ }^{34}$ More importantly for intestinal exposure to carcino- gens, heterocyclic amines and their hydroxylamine metabolites, which are substrates for $\mathrm{NAT}^{5}$ are generated during the cooking of "well done" meat. ${ }^{6-8}$ NAT also metabolises arylamine drugs including 5-aminosalicylic acid (5-ASA). ${ }^{19}$

There are two NAT isozymes in humans, NAT1 and NAT2, both of which are now known to be polymorphic. ${ }^{10}$ There has been a continued discussion on the role of NAT polymorphism in determining susceptibility to colorectal cancer. Most of the investigations of NAT as a susceptibility factor in colorectal cancer have involved NAT2 polymorphism ${ }^{11}$ as it has been better characterised than NAT1 polymorphism. The situation with colorectal cancer is not as clear cut as in bladder cancer where slow acetylators at the NAT2 locus are undoubtedly at increased risk. ${ }^{12}{ }^{13}$ In colorectal cancer the role of NAT2 polymorphism has been controversial. NAT2 fast acetylators have been suggested to be at increased risk of colorectal cancer, ${ }^{5}{ }^{14}$ but others ${ }^{15}$ disagree with these findings. It has been suggested that fast NAT2 alleles are of increased importance as susceptibility factors in association with a particular $N A T 1$ allele-the $N A T 1^{\star} 10$ allele ${ }^{14}$-as detected by a mutation in the polyadenylation site but the association of the mutation with colorectal cancer has been disputed. ${ }^{16}$ Recently it has been shown that alleles genotyped as $N A T 1^{\star} 10$ by detection of the mutation in the polyadenylation $\operatorname{site}^{1417}$ are likely to include examples of individuals who also have a $G$ to $A$ mutation converting amino acid 187 from an arginine to a glutamine. ${ }^{18} 19$ This mutation affects the activity of NAT1 such that the $N A T 1^{\star} 10$ variant has a reduced level of activity. The newly identified $N A T 1^{\star} 10$ variant has been named $N A T 1^{\star} 14^{18} 20$ and a method for genotyping NAT1 which allows detection of NAT $1^{\star} 14$ has been described..$^{18}$

Both NAT1 and NAT2 activities have been described in intestine ${ }^{921}$ and these enzymes will catalyse the $\mathrm{N}$-acetylation of arylamine drugs, such as 5-ASA; arylhydoxylamines are generated by cytochrome P450 catalysed oxidation of arylamines in cooked meat. ${ }^{7}$ The role of NAT in carcinogenesis is thought to be important in catalysing the formation of the "ultimate carcinogen", an $\mathrm{N}$-acetoxyester which reacts with DNA. ${ }^{22}$ It has been suggested using expressed human NAT1 and NAT2 that the human NAT2 enzyme is more active with heterocyclic amines as substrates than is NAT $1 .^{5} \mathrm{It}$ has also been shown, using heterologously expressed human NAT1 and NAT2, that the enzymes can catalyse both activation 
a

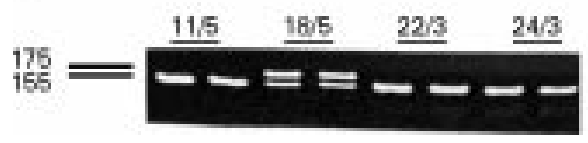

Figure 1 Identification of NAT1 alleles by PCR and restriction enzyme digestion with BsaOI. Template DNA was amplified with the primer pair N539 (sense) and N714 (antisense) and then digested with BsaOI. The wild type allele ( $G$ at position 560 of coding sequence) $\mathrm{NAT}^{\star}{ }_{4}$ is digested to a $155 \mathrm{~kb}$ fragment. The mutant allele $\left(N A T 1^{\star} 14\right)$ is not digested by BsaOI and the band is $175 \mathrm{~kb}$. The products were separated on a 4\% Metaphor agarose gel. (a) genomic DNA as template. (b) Tracks 1 and 2 show genomic DNA of individual 22/3 as template. Tracks 3 and 4: the template was the product of a "nested PCR" using initially allele specific amplification (N539 and N1110b as primers) with genomic DNA from individual 18/5. The primers are described in table 1.

(O-acetylation) and deactivation $(\mathrm{N}-$ acetylation) reactions of heterocyclic amines and amines with a carbon only aromatic ring. ${ }^{23}$ The reaction rate depends on the isozyme, the reaction, and the substrate. Therefore interindividual variation in intestinal activities of NAT1 and NAT2 is likely to affect the range of carcinogenic compounds generated in situ.

$N A T$ genes are encoded on $8 \mathrm{p} 22^{24}$ - a region which is frequently deleted in many tumours including colorectal tumours. ${ }^{25}$

The NAT1 isozyme is likely to be more important in 5-ASA acetylation than NAT2 from comparison of the activities of the expressed proteins. The NAT1 isoenzyme has been shown to be 19000 -fold more active with 5-ASA than the NAT2 isoenzyme. ${ }^{23}$

In view of the potential role of NAT in intestinal cancer and in metabolism of 5-ASA, which is used for local application in inflammatory bowel disease, it is important to establish the pattern of expression in normal intestine. We have therefore used a combination of enzymic and immunological methods to investigate NAT expression in human intestine obtained from organ donors.

\section{Methods}

TISSUE SAMPLES

Human intestines and livers were removed from adult organ donors who were victims of vehicular or cerebrovascular accidents. Briefly, the intestine was rinsed with ice cold $0.9 \%$ $\mathrm{NaCl}$ to remove particulate debris. The liver was perfused with $0.9 \% \mathrm{NaCl}$. Intestinal tissue was sectioned into $30 \mathrm{~cm}$ long portions and four equally spaced sections from the duodenum to the ileocaecal junction were numbered as sections 1-4. Equally spaced sections from the caecum to the rectum were numbered 5-8. The portions were placed in numbered polythene bags and the tissue was frozen in liquid nitrogen and then stored at $-70^{\circ} \mathrm{C}$. Intestinal tissue was defrosted on an ice cold glass plate. The mucosa was scraped from the outer muscle layer using a metal spatula and transferred to four volumes of ice cold homogenisation buffer, $250 \mathrm{mM}$ sucrose, $100 \mathrm{mM}$ phosphate (pH 7.4), $1 \mathrm{mM}$ EDTA, and $1 \mathrm{mM}$ DTT, with the proteinase inhibitor leupeptin $(1 \mu \mathrm{g} / \mathrm{ml})$. Liver was defrosted at room temperature and sliced into small pieces prior to homogenisation as above. Cytosols were prepared by centrifugation. The cytosolic fraction was the supernatant after centrifugation of the ho- mogenate $(10000 \mathrm{~g}$ for 20 minutes, followed by $100000 \mathrm{~g}$ for 60 minutes at $4^{\circ} \mathrm{C}$ ). Aliquots $(1 \mathrm{ml})$ of cytosols were made and stored in liquid nitrogen apart from transportation on dry ice from Seattle to Oxford. Samples remained frozen and were returned to liquid nirogen immediately on arrival.

ENZYMIC ASSAYS

The $N$-acetylation of $100 \mu \mathrm{M} p$-aminobenzoic acid and $1000 \mu \mathrm{M}$ sulphamethazine were used as probes for human NAT1 and NAT2 activities respectively. Cytosols $(40 \mu \mathrm{g}$ protein) were incubated for five or 10 minutes at $37^{\circ} \mathrm{C}$ with either $p$-aminobenzoic acid or sulphamethazine in the presence of $100 \mu \mathrm{M}$ acetyl CoA and an acetyl carnitine based recycling system ${ }^{26}$ in a total volume of $90 \mu \mathrm{l}$. Reactions were stopped by the addition of $10 \mu \mathrm{l}$ of $15 \%$ perchloric acid. Acetylated metabolites in the supernatant after centrifugation $(20000 \mathrm{~g}$ for five minutes at $4^{\circ} \mathrm{C}$ ) were measured by high pressure liquid chromatography with ultraviolet detection $(266 \mathrm{~nm})$, as previously described. ${ }^{27}$

ANTIBODIES

Antibodies recognising NAT1 and NAT2 (called 177), specific for NAT1 (called 184), or specific for NAT2 (called 4768) were prepared as described previously. ${ }^{28}$ Western blots were carried out as described previously using a dilution of the first antibody of $1 / 500$ to $1 / 12000$. The second antibodies (goat antirabbit immunoglobulin) were coupled with horseradish peroxidase and were used at a dilution of $1 / 10000$. Slides bearing $10 \mu \mathrm{m}$ thick sections of formalin fixed, paraffin wax embedded human small intestine and colon were subjected to immunohistochemical staining using the Vecastain Elite ABC peroxidase (Universal) kit (Vector Labs, Peterborough, UK). Endogenous peroxidase activity was inhibited using $1 \%$ (vol/vol) $\mathrm{H}_{2} \mathrm{O}_{2}$ in $15 \mathrm{mM}$ Tris- $\mathrm{HCl}$, pH 7.5 containing $140 \mathrm{mM} \mathrm{NaCl}$ (TBS); sections were incubated with rabbit antiserum or preimmune serum at $4^{\circ} \mathrm{C}$ overnight and three 10 minute washes were performed between each antibody incubation. Rabbit antisera were diluted in TBS containing $1 \%$ normal horse serum. Following immunostaining, sections were counterstained with Gill's haematoxylin and dehydrated. Coverslips were attached with DPX mountant.

\section{GENOTYPING}

DNA was prepared from homogenised pieces of intestine by extraction with phenol and chloroform..$^{13}$ NAT2 genotype was determined by the method of Risch et $a l^{13}$ and NAT1 genotype was determined by a modification of the method described by Payton and Sim. ${ }^{18}$ Briefly, NAT1 alleles were initially identified by MboII restriction fragment length polymorphism of polymerase chain reaction (PCR) products. ${ }^{14}$ The presence of a mutation ( $G$ in wild type for A in mutant alleles) at nucleotide 560 in the coding region of NAT1 was detected by BsaOI restriction fragment length polymorphism of the PCR products obtained using primer N539 
b

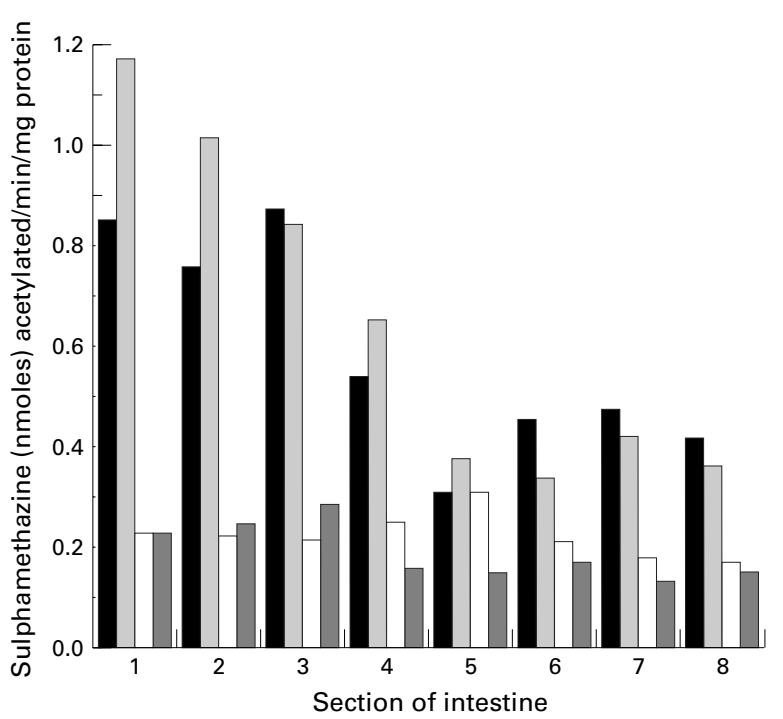

11/5:1

18/5:1

$\square$ 22/3:1

$\square$ 24/3:1

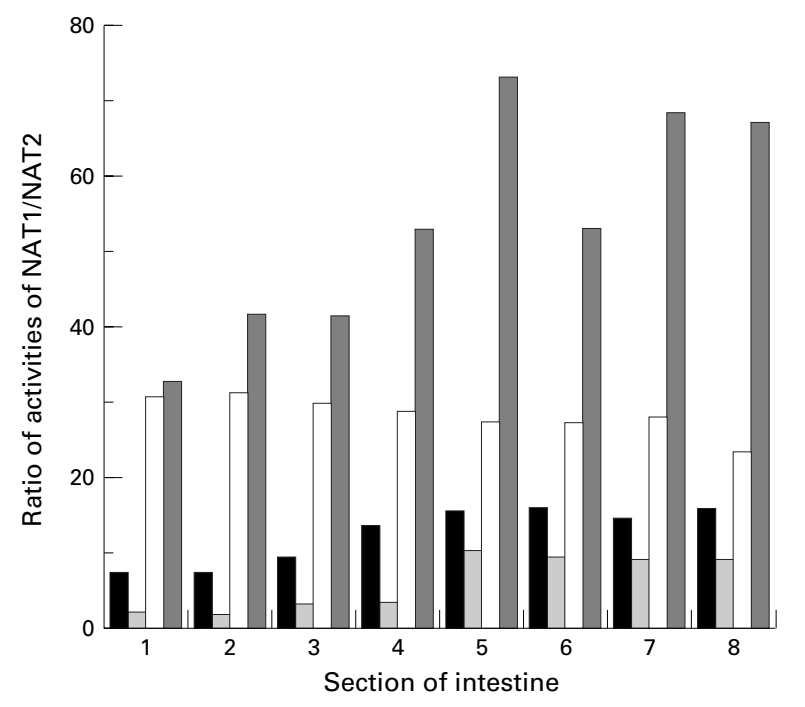

Figure 2 Detection of NAT enzymic activity in different sections along the length of human intestine. (a) Enzymic acivity of NAT1 was determined in cytosols with p-aminobenzoic acid; (b) NAT2 activity was determined with sulphamethazine. (c) Ratios of activities of NAT1 to NAT2. Samples 1-8 correspond to sections along the length of the intestine from the duodenum (1) to the rectum (8). as the sense primer with primer $\mathrm{N} 714$ as the antisense primer (table 1). Allele specific PCR using primer N539 as sense primer with $\mathrm{N} 1110 \mathrm{~b}$ as antisense primer was used to confirm the mutant allele as $N A T 1^{\star} 14$. Amplication conditions and restriction enzyme digests were as defined previously. ${ }^{18}$

\section{Results}

GENOTYPING AND INTESTINAL ACTIVITY OF $N$-ACETYLTRANSFERASES

Table 2 illustrates the genotype of NAT1 and NAT2 in the four intestinal samples. The activities of NAT1 and NAT2 were determined using $p$-aminobenzoic acid and sulphamethazine, respectively, as substrates. The relation between the phenotype of NAT2 determined from the genotype and the measured activities of samples with sulphamethazine as substrate is as would be expected. Two of the samples were determined as homozygous for slow acetylator alleles and these samples had the lowest activi- ties with sulphamethazine. The sample which was determined as being homozygous fast for NAT2 had the highest NAT2 activity and the individual who was determined as having one fast and one slow NAT2 allele had an intermediate level of activity. The correlation of NAT2 activity in intestinal samples towards sulphamethazine with NAT2 type has been commented on by Ilett et al. ${ }^{21}$ As in the present study, Ilett et al found the level of NAT1 activity to be greater than the level of NAT2 activity.

The NAT1 activities of three of the samples in the present study determined with $p$-aminobenzoic acid as substrate were similar and these samples were all genotyped as being homozygous for the wild type $N A T 1^{\star} 4$ allele. One individual had a lower level of NAT1 activity and was genotyped as $1^{\star} 4,1^{\star} 14$ (fig 1 ). It has previously been shown that a mutation at nucleotide 560, as is found in allele $N A T 1^{\star} 14$, which results in the substitution of an arginine residue in the wild type NAT1 at amino acid 
a

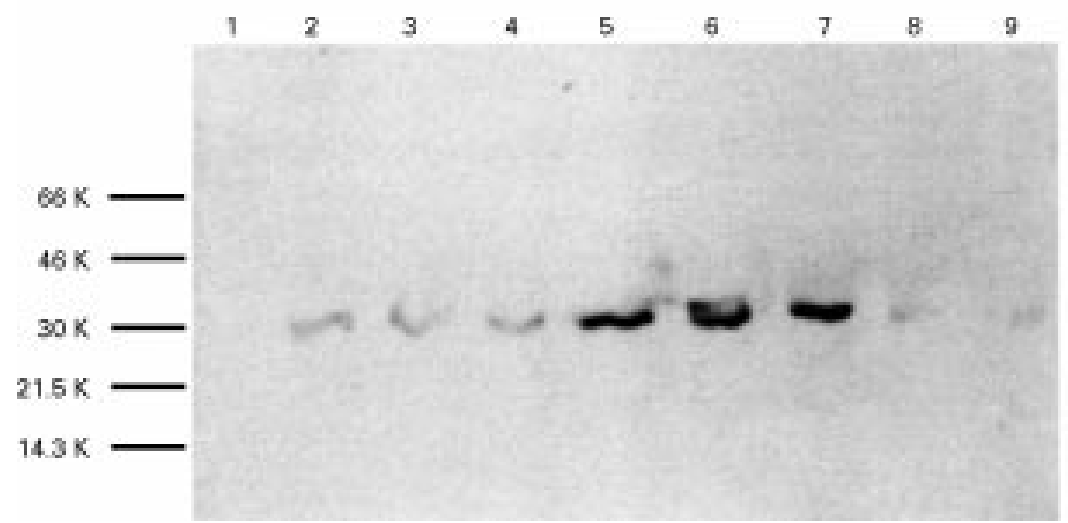

b

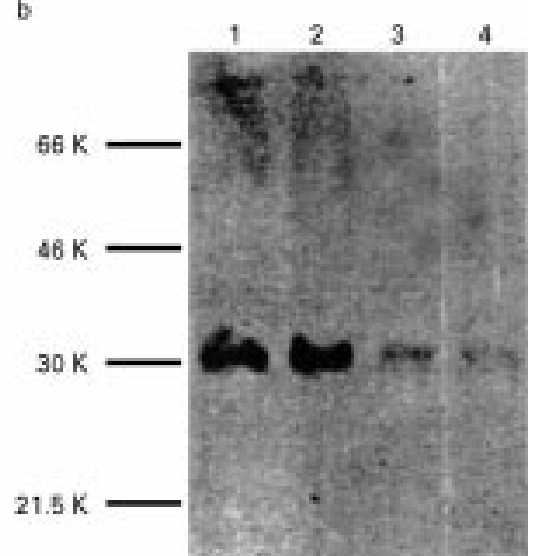

Figure 3 Expression of NAT1 in human intestine detected by western blotting. (a) Samples of mucosal cytosol along the length of human intestine from individual 22/3 were prepared and analysed by polyacrylamide gel electrophoresis and western blotting (tracks 2-9). The sample in track 2 is from the most proximal section (corresponding to 1 in fig 2) and the sample in track 9 is from the rectal end. Track 1 contained the molecular weight markers which are not detected by the antiserum. (b) Samples (52 $\mathrm{mg}$ total protein) from the cytosols with maximum activity from individual $22 / 3$ (tracks 1 and 2) and from individual 18/5 (tracks 3 and 4) were analysed. The first antibody was antiserum 184 used at a dilution of 1 in 1000 and the second antibody was goat antirabbit immunoglobulin at a dilution of 1 in 10 000. The molecular weights were determined from prestained markers run on the same gel.
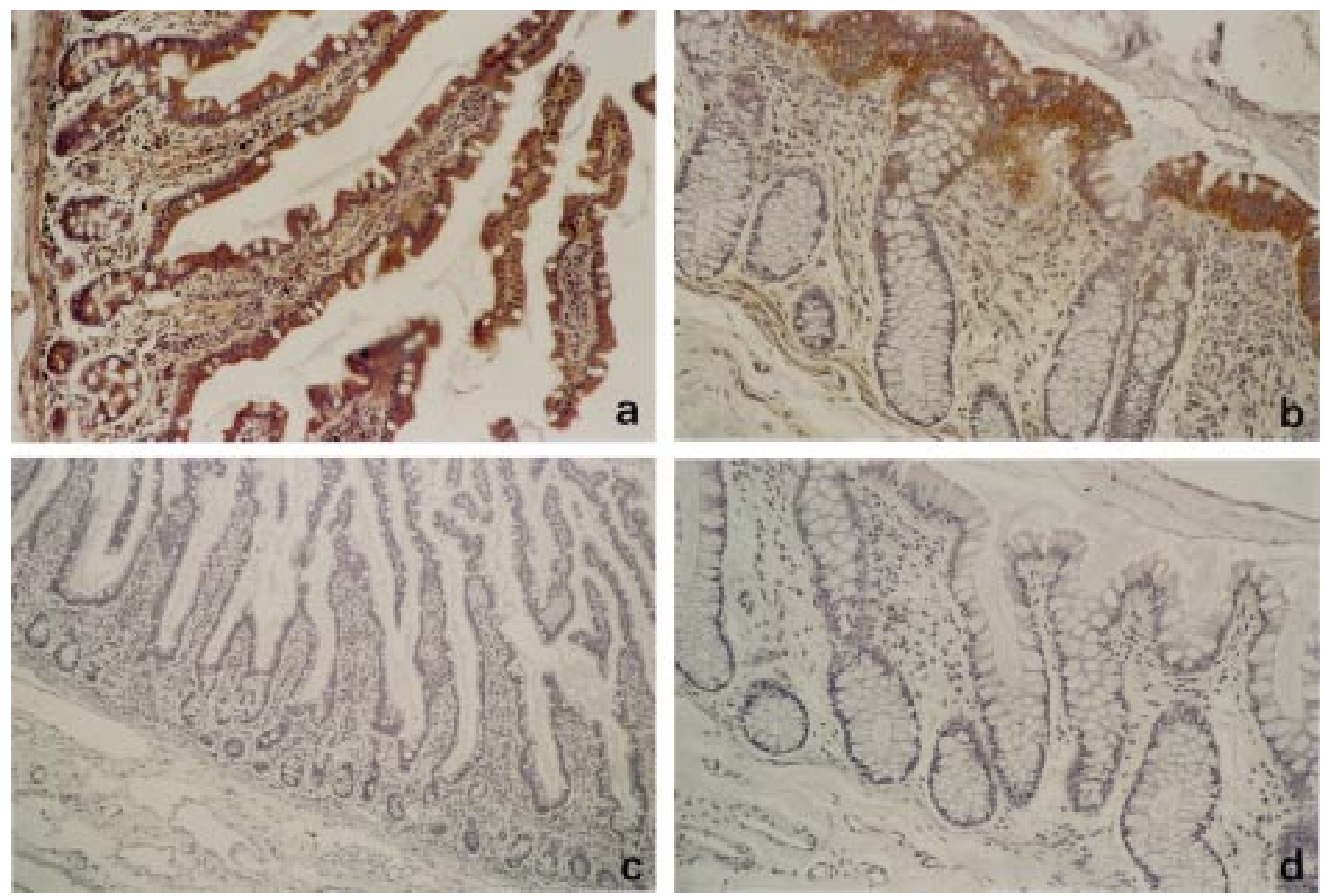

Figure 4 Expression of NAT in human intestine detected by immunohistochemistry with antiserum 177. Sections of duodenum (a,c) and colon (b,d) were stained with antiserum 177 (diluted 1 in 3000) (a,b) or the corresponding dilutions of preimmune serum (c,d). Intestine from individuals 18/5 (a,c) and $24 / 3(b, d)$ are shown. Original magnification: $(c) \times 100 ;(a, b, d) \times 200$.

residue 187 for a glutamine in the mutant, is associated with a low level of NAT1 activity. ${ }^{18} 20$ The identification of this mutation in an individual with a low level of NAT1 activity in intestine further confirms the relation between this allele and reduced NAT1 activity.

ACTIVITY ALONG THE LENGTH OF HUMAN INTESTINE

The distribution of NAT 1 and NAT2 along the length of the intestine was measured in two ways. It was measured by determining the activity of NAT1 with $p$-aminobenzoic acid and the activity of NAT2 with sulphamethazine. NAT1 activity was found in all sections of intestine. In three of the individuals the activity was measured maximally at the region between the small and large intestine (fig 2a). However with sample $18 / 5$, showing the lowest level of NAT1 activity, there was an increase in activity towards the distal end of the intestine (fig 2a). The distribution of activity in samples along 

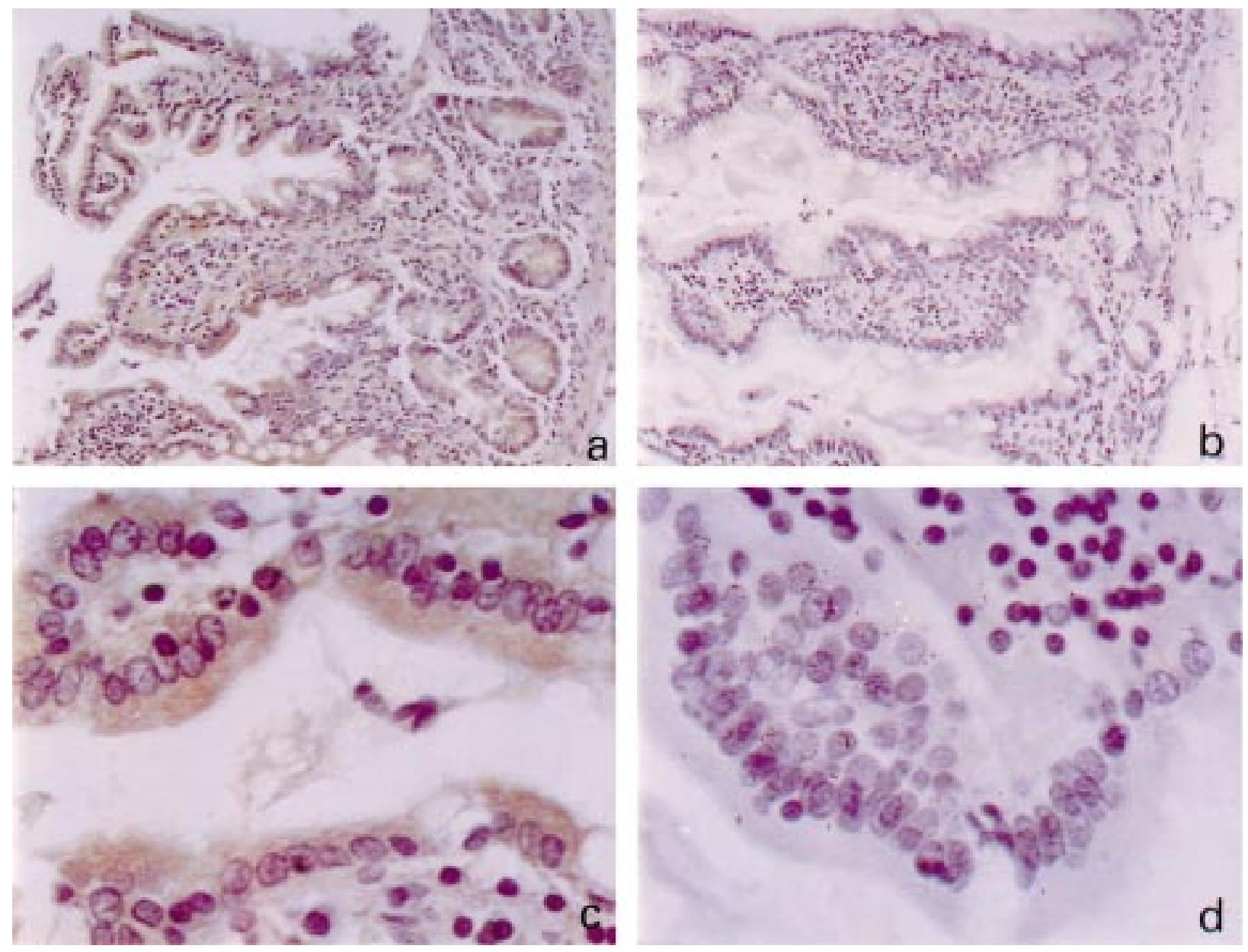

Figure 5 Expression of NAT1 in human intestine detected by immunohistochemistry with antiserum 184. Small intestine from individual 22/3 was stained with antiserum 184 (diluted 1/800) (a,c) or with the corresponding dilution of preimmune serum (b,d). Original magnification: (a,b) $\times 200$; (c,d) $\times 1000$.

the length of the intestine was matched by the NAT1 antigenic activity detected by western blotting of the same amounts of protein from each section of intestine (fig 3). The antiserum identifies a band at around 33000 molecular weight corresponding to the expected molecular weight of NAT $1 .^{10}$

The distribution of NAT2 along the length of the intestine showed a decrease from the proximal to the more distal sections of around twofold in the two fast acetylator individuals (fig $2 \mathrm{~b}$ ). If the ratios of NAT1 activity to NAT2 activities are compared along the length of the intestine it is clear that there is considerable interindividual variation within this small group of individuals (fig 2c). Individuals 24/3 and $22 / 3$ are both slow acetylators for NAT2 and have two wild type alleles for NAT1. The NAT1 activities of both of these individuals compared with NAT2 activities show much higher relative NAT1 activities in intestine. This is particularly notable for individual $24 / 3$ who has 50 to 70 times more NAT1 activity than NAT2 activity in the lower sections of intestine. The ratio of NAT1 activity to NAT2 activity is more similar in the two individuals who are homozygous (11/5) or heterozygous $(18 / 5)$ fast acetylators at the NAT2 locus. The intestine of individual 18/5, who carries the mutant $N A T 1^{\star} 14$ allele, has a maximum of 10 times more NAT1 than NAT2 activity, but again the biggest difference between the two enzymic activities is in the colon rather than the small intestine. The level of expression of NAT activity in the different individuals is reflected in the antigenic activities detected in samples from the same region of the gut containing the same amount of total protein (fig 3). In both individuals a band appears at around 33000 molecular weight (the expected molecular weight of NAT1), indicating that the lower activity of $18 / 5$ is not due to proteolytic degradation of NAT 1 protein. It seems that in the individual with the $N A T 1^{\star} 14$ allele there is less protein detectable by the NAT1 specific antiserum, 184. It is unlikely that this is due to a difference in the recognition of the mutant NAT1 by this antibody as the antibody recognises the C-terminal peptide of NAT1 which is the same in the isoenzymes encoded by both the $N A T 1^{\star} 4$ and $N A T 1^{\star} 14$ alleles. It would seem that the amount of NAT protein in the individual with the $N A T 1^{\star} 14$ allele is less. Site directed mutagenesis studies are required to confirm whether the protein product of the mutant allele with arginine 187 mutated to glutamine is active or not. 
Table 1 Primers used for detecting NAT1 ${ }^{\star} 14$ allele

\begin{tabular}{|c|c|c|c|}
\hline Primer & Sequence 5'-3' & $\begin{array}{l}\text { Annealing } \\
\text { temperature } \\
\left({ }^{\circ} \mathrm{C}\right)\end{array}$ & Comment \\
\hline Sense N539 & ${ }^{539} \mathrm{TCC}$ TAG AAG ACA GCA A $\underline{C G} A C C^{559}$ & & Bsa0I site in alleles with $\mathrm{G}^{560}$ \\
\hline Antisense N1110b & ${ }^{1110}$ GGC CAT CTT TAA AAT ACA TTT $T^{1089}$ & 54 & Specific for alleles NAT $1 \star 10$ and NAT $1{ }^{\star} 14$ \\
\hline N714 & ${ }^{714}$ GTG AAG CCC ACC AAA CAG ${ }^{697}$ & 52 & \\
\hline
\end{tabular}

The numbers refer to the corresponding nucleotides in the coding region of NAT1. The underlined nucleotides have been mutated from the NAT1 sequence of the wild type to introduce a Bsa0I site (indicated by italics) in all alleles except NAT $1^{\star} 14$ and others without a G at $560 .^{18}$ The sense primer and $\mathrm{N} 1110 \mathrm{~b}$ as antisense primer were used with genomic DNA as template. The product was used as template with N539 with N714 as antisense primer ("nested" PCR). Primers N539 and N714 were used to amplify genomic DNA as template also as has been described. ${ }^{18}$

Table 2 Genotypes of NAT in intestinal samples

\begin{tabular}{|c|c|c|c|c|c|}
\hline \multirow[b]{2}{*}{ Sample } & \multirow[b]{2}{*}{ NAT1 } & \multirow[b]{2}{*}{$\begin{array}{l}\text { PABA activity } \\
\text { (nmol/min/mg protein) }\end{array}$} & \multicolumn{2}{|l|}{$N A T 2$} & \multirow[b]{2}{*}{$\begin{array}{l}\text { SMZ activity } \\
\text { (nmol/min/mg protein) }\end{array}$} \\
\hline & & & Genotype & Phenotype & \\
\hline $\mathrm{A}(11 / 5)$ & $1^{\star} 4,1^{\star} 4$ & $6.1(0.2)$ & $2^{\star} 4,2^{\star} 5 \mathrm{~B}$ & FS & $0.85(0.01)$ \\
\hline B $(18 / 5)$ & $1^{\star} 4,1^{\star} 14$ & $2.1(0.1)$ & $2^{\star} 4,2^{\star} 4$ & $\mathrm{FF}$ & $1.18(0.03)$ \\
\hline$C(22 / 3)$ & $1^{\star} 4,1^{\star} 4$ & $6.9(0.3)$ & $2 \star 5 B, 2 \star 6 A$ & SS & $0.22(0.02)$ \\
\hline D $(24 / 3)$ & $1^{\star} 4,1^{\star} 4$ & $7.5(0.1)$ & $2 \star 5 \mathrm{~B}, 2 \star 5 \mathrm{~B}$ & SS & $0.23(0.01)$ \\
\hline
\end{tabular}

The names of alleles are as described in Vatsis et al. ${ }^{10}$ The phenotype of NAT2 has been determined from the genotype and is shown to indicate fast alleles as ' $F$ ' and slow alleles as ' $S$ '. Activities with sulphamethazine (SMZ) or $p$-aminobenzoic acid (PABA) are the values for the most proximal section of intestine in each case and are the mean (SD) of four individual estimations.

CELLULAR DISTRIBUTION OF NAT

Antiserum 177 which recognises NAT1 and NAT2 and antiserum 184 raised against a C-terminal peptide of NAT1 have been shown previously to be specific. ${ }^{28}$ Paraffin wax sections from the small intestine and colon of all four individuals were stained with either 177 (fig 4) or 184 (fig 5). The pattern of staining in all four individuals was very similar to the representative samples shown in fig 4 . In the small intestine, staining was observed in the epithelial cells along the whole length of the villus, from the tip to the crypt of Lieberkuhn (fig $4 \mathrm{a}$, fig 5a). The staining was cytoplasmic and was seen in all epithelial cell types. In goblet cells the antibody seems to be excluded from the mucous inclusions which are visible as transparent areas within the stained cytoplasm. In the colon, however, staining was restricted to the epithelial cells at the luminal surface, the crypts being devoid of staining (fig $4 \mathrm{~b}$ ). The most intense staining was found at the luminal tip of the columnar epithelial cells. As in the small intestine, the antibody seemed to be excluded from the mucous inclusions of goblet cells. No staining was detected in either the small intestine or the colon when preimmune rabbit serum was used instead of antiserum 177 or 184 .

The existence of NAT in villi has also been identified by microdissection of villi from the underlying muscle. A western blot showed no band for the same amount of muscle protein whereas the villi showed a band corresponding to NAT1 (fig 6). These results agree with a previous preliminary report ${ }^{29}$ which illustrated that the NAT1 activity in cytosol from mucosa of small intestine was six times greater than the activity associated with a muscle cytosol, while no NAT2 activity could be detected in the muscle cytosol.

\section{Discussion}

The detection of NAT activity in intestine was established by the early work of Jenne ${ }^{9}$ who showed using isoniazid that intestinal mucosa and liver had equivalent specific activities of NAT. Isoniazid is now known to be an NAT2 substrate. ${ }^{30}$ However, it seems that NAT activity in human intestine is mainly due to NAT1. At the time when Jenne carried out his studies, although he used $p$-aminosalicylate, now known to be a NAT1 substrate, ${ }^{23}$ he did not report on activities of individual intestinal homogenates with both isoniazid and $p$-aminosalicylate. It is not possible to compare his data with the present comparison of NAT1 and NAT2 activities in human intestine. Jenne suggested that the activities of NAT2 in liver and intestine were very similar. In a preliminary report ${ }^{31}$ for three individuals where NAT2 in liver and intestine were measured it was found that NAT2 specific activities in liver were either two- to threefold higher than in intestine in fast NAT2 acetylators or of a comparable value in slow NAT2 acetylators. Comparison of

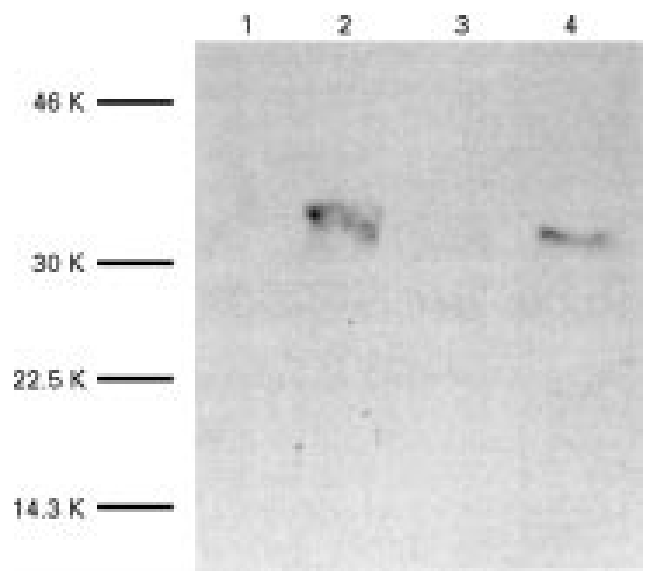

Figure 6 Determination of NAT expression in villi and muscle by western blotting. Villi were microdissected from muscle samples of small intestinal samples (corresponding to track 4 of fig 3a) and large intestine (corresponding to track 7 of fig 3a). The mucosa or muscle mixtures $(40 \mu \mathrm{g}$ total protein of each) were analysed by gel electrophoresis and western blotting using antibody 184 as above. Tracks 2 and 4 show villi and tracks 1 and 3 show muscle. Tracks 1 and 2 are small intestine and tracks 3 and 4 are large intestine. 
activities of NAT1 and NAT2 in intestines of the same individual has shown in all samples that there is a much higher specific activity of NAT1 than NAT2. This is found in both fast and slow acetylators although the difference between the two activities is much more notable in slow acetylators at the NAT2 locus. These results are fully in agreement with the studies of Ilett et al. ${ }^{21}$ The present results also show that the genotype of NAT1 affects the activities of NAT1 detected in intestine. The detection of NAT1 along the length of intestine suggests that as far as the colon is concerned the activity of NAT1 is likely to be extremely important in generating compounds from aromatic arylamines or hydroxylamines which are likely to have a role in colon carcinogenesis. ${ }^{6-8}$ The interindividual variation even in the four samples which were available indicates that genotyping for NAT1 in colorectal cancer has a sound biochemical basis. The lack of a clear correlation of NAT2 type with colorectal cancer susceptibility ${ }^{15}$ may be due to the low level of NAT2 which is present in colon in comparison with NAT1.

Animal studies have also shown that NAT is present in intestine in hamster, ${ }^{32}$ mouse, ${ }^{32-34}$ where it has been localised to the villi of the small intestine, ${ }^{33}$ and rat. $^{32}$ In these rodents there is evidence for a difference in expression of individual NAT isozymes along the length of the intestine. Therefore the understanding of the expression of human NAT isoenzymes along the length of the intestine together with comparison of the substrate specificities of the NAT isoenzymes of different species will allow the establishment of sensible models for understanding human colon carcinogenesis ${ }^{35}$ and may serve as an additional means of validating animal models of inflammatory bowel disease. ${ }^{36}$

The identification of NAT in epithelial cells may be important because susceptibility factors in tumour development include preexisting adenomas. ${ }^{37}$ Ulcerative colitis or Crohn's disease are also associated with epithelial dysplasia and have been considered to be precursor lesions, rather than predisposing factors for colon carcinoma. In the inflammatory bowel disorders, the methods are available to determine whether NAT 1 expression is altered. NAT1 polymorphism may also have a role in the therapeutic response or adverse effects to $5-\mathrm{ASA}^{38}$ and the epithelial localisation of NAT1 which is shown here to be the most active NAT in colon should be borne in mind when designing other drugs active in inflammatory bowel disease.

We are grateful to Aileen Watson and Susan Rolls for excellent technical assistance, to Gamini Chandrasena for advice and help in processing human intestine, to Mike Ball for expert assistance in processing human intestine, to Mike Ball for expert assistance
with histology and photomicrography, and to Dr Bryan Warren with histology and photomicrography, and to Dr Bryan Warren for helpful discussions. The Oxford work was supported by the
Association for International Cancer Research and the Wellcome Trust. The work was also funded in part by grants come Trust. The work was also funded
NIHA127664 and NIHDK41978 (JDU).

1 Weber WW, Hein DW. N-acetyltransferase pharmacogenetics. Pharmacol Rev 1985;37:25-80.

2 Sim E, Stanley LA, Risch A, et al. Xenogenetics in multifactorial disease susceptibility. Trends Genet 1995;11:509-12.
3 Ambrosone CB, Freudenhein JL, Graham S, et al. Cigarette smoking, N-acetyltransferase 2 genetic polymorphisms and smoking, $\mathrm{N}$-acetyltransferase 2 genetic polymor
breast cancer risk. $¥ A M A$ 1996;276:1494-501.

4 Vineis P, Bartsch H, Caporaso N, et al. Genetically based $\mathrm{N}$-acetyltransferase metabolic polymorphism and low level environmental exposure to carcinogens. Nature 1994;369: 154-6.

5 Minchin RF, Kadlubar FF, Ilett KF. Role of acetylation in colorectal cancer. Mutat Res 1993;290:35-42.

6 Sugimura T. Carcinogenicity of mutagenic heterocyclic amines formed during the cooking process. Mutat Res 1985;150:33-41.

7 Gooderham NJ, Murray S, Lynch AM, et al. Heterocyclic amines: evaluation of their role in diet associated human cancer. Br 7 Clin Pharmacol 1996;42:91-8.

8 Josephy PD. Molecular toxicology. New York: Oxford University Press, 1997:352-63.

9 Jenne JW. Partial purification and properties of the isoniazid transacetylase in human liver. Its relationship to the acetylation of p-aminosalicylic acid. f Clin Invest 1965;44: 1992-2002.

10 Vatsis KP, Weber WW, Bell DA, et al. Nomenclature for $\mathrm{N}$-acetyltransferases. Pharmacogenetics 1995;5:1-36.

11 Wohlleb JC, Hunter CF, Blass B, et al. Aromatic amine acetyltransferase as a marker for colorectal cancer: environmental and demographic associations. Int $f$ Cancer 1990;46:22-30.

12 Cartwright RA, Glashan RW, Rogers HJ, et al. Role of $\mathrm{N}$-acetyltransferase phenotypes in bladder carcinogenesis: pharmacogenetic epidemiological approach to bladder cancer. Lancet 1982;ii:842-6.

13 Risch A, Wallace DMA, Bathers S, et al. Slow N-aceylation genotype is a susceptibility factor in occupational and smoking related bladder cancer. Hum Mol Genet 1995;4: 231-6.

14 Bell DA, Stephens EA, Castranio T, et al. Polyadenylation polymorphism in the acetyltransferase 1 gene (NAT1) increases risk of colorectal cancer. Cancer Res 1995;55: 3537-42.

15 Probst-Hensch NM, Haile RW, Ingles SA, et al. Acetylation polymorphism and prevalence of colorectal adenomas. polymorphism and prevalen

16 Probst-Hensch NM, Haile RW, Li DS, et al. Lack of association between the polyadenylation polymorphism in the NAT1 (acetyltransferase 1) gene and colorectal adenomas. Carcinogenesis 1996;17:2125-9.

17 Bell DA, Badawi AF, Lang NF, et al. Polymorphism in the $\mathrm{N}$-acetyltransferase 1 (NAT1) polyadenylation signal: association of NAT $1{ }^{\star} 10$ allele with higher N-acetylation activity in bladder and colon tissue. Cancer Res 1995;55: 5226-9.

18 Payton MA, Sim E. Genotyping human arylamine N-acetyltransferase type 1 (NAT1): the identification of a $\mathrm{N}$-acetyltransferase type 1 (NAT1): the identic
novel mutation. Biochem Pharmacol (in press).

19 Hubbard AL, Harrison D, Wylie A. NAT1 genotyping in Hubbard AL, Harrison D, Wylie A. NAT
colorectal cancer. Gut 1997;41:229-35

20 Grant DM, Hughes NC, Janezic SA, et al. Human acetyltransferase polymorphisms. Mutat Res 1997;376:6170

21 Ilett KF, Ingram DM, Carpenter DS, et al. Expression of monomorphic and polymorphic $\mathrm{N}$-acetyltransferases in human colon. Biochem Pharmacol 1994;47:914-7.

22 Flammang TJ, Westra JG, Kadlubar FF, et al. DNA adducts formed from the probable proximate carcinogen, N-hydroxy-3,2'-dimethyl-4-aminobiphenyl, by acid catalysis or S-acetyl coenzyme A-dependent enzymatic esterificasis or S-acetyl coenzyme A-dependen
tion. Carcinogenesis 1985;6:251-8.

23 Hein DW, Doll MA, Rustan TD, et al. Metabolic activation and deactivation of aryalmine carcinogens by recombinant human NAT1 and polymorphic NAT2 acetyltransferases. Carcinogenesis 1993;14:1633-8.

24 Matas N, Thygesen P, Stacey M, et al. Mapping AAC1, AAC2 and AACP, the genes for the carcinogen metabolising enzymes, arylamine $\mathrm{N}$-acetyltransferases, on human chromosome $8 \mathrm{p} 22$, a region frequently deleted in tumours. Cytogenet Cell Genet 1997;77:290-5.

25 Farrington SM, Cunningham C, Boyle SM, et al. Detailed physical and deletion map of $8 \mathrm{p}$ with isolation of YAC clones from tumour suppressor loci involved in colorectal cancer. Oncogene 1996;12:1803-8.

26 Mattano SS, Weber WW. Kinetics of arylamine $\mathrm{N}$-acetyltranferase in tissues from rapid and slow acetylator -acetyltranferase in tissues from

27 Hickman D, Palamanda JR, Unadkat JD, et al. Enzyme kinetic properties of human recombinant arylamine kinetic properties of human recombinant arylamine
$\mathrm{N}$-acetyltransferase 2 allotypic variants expressed in $\mathrm{N}$-acetyltransferase 2 allotypic variants expressed
Eschericia coli. Biochem Pharmacol 1995;50:697-703.

28 Stanley LA, Coroneos E, Cuff R, et al. Immunochemical detection of arylamine $\mathrm{N}$-acetyltransferase in normal and neoplastic bladder. $\mathcal{F}$ Histochem Cytochem 1996;44:105967.

29 Hickman D, Chandrasena G, Unadkat JD. Longitudinal distribution of arylamine $\mathrm{N}$-acetyltransferases along the human small intestine. 4th International Society for the Study of Xenobiotics (ISSX) Meeting; 1995 Aug 27-31; Seattle. Seattle: ISSX, 1995:250.

30 Deguchi T, Mashimo M, Suzuki T. Correlation between acetylator phenotypes and genotypes of polymorphic arylamine $\mathrm{N}$-acetyltransferase in human liver. $\mathrm{f}$ Biol Chem 1990;265:12757-60.

31 Hickman D, Chandrasena G, Patil SD, et al. Arylamine $\mathrm{N}$-acetyltransferase activities in human liver and intestine. 8th Japanese-American Conference on Pharmacokinetics 
and Pharmaceutics; 1996 July 28-30; Seattle. JapaneseAmerican Conference on Pharmacokinetics, 1996:28.

32 Ware JA, Svensson CK. Longitudinal distribution of arylamine $\mathrm{N}$-acetyl transferase in the intestine of the hamster, mouse and rat. Evidence for multiplicity of $\mathrm{N}$-acetyltransferase in the intestine. Biochem Pharmacol 1996;52:1613-20.

33 Stanley LA, Mills IG, Sim E. Localisation of polymorphic $\mathrm{N}$-acetyltransferase type II (NAT2) in tissues of inbred mice. Pharmacogenetics 1997;7:121-30.

34 Chung JG, Levy GN, Weber WW. Distribution of 2-aminofluorene and p-aminobenzoic acid N-acetyl transferase activity in tissues of $\mathrm{C} 57 \mathrm{Bl} / 6 \mathrm{~J}$ Rapid and B6.ANat ${ }^{\mathrm{s}}$ slow acetylator congenic mice. Drug Metab Dispos 1993;21:1057-63.

35 Levy GN, Rodgers L, Weber WW. Effects of heredity on response to drugs and environmental chemicals: construction of rodent models. Chem Res Toxicol 1996;9:1217-24. 36 Warren BF, Watkins PE. Animal models of inflammatory

7 Hill MJ. Aetiology of colorectal cancer: current concepts. Baillieres Clin Gastroenterol 1989;3:567-92.

38 Schreiber S, Hamling J, Zehnter E, et al. Renal tubular dysfunction in patients with inflammatory bowel disease treated with aminosalicylate. Gut 1997;40:761-6. 